\title{
Visualizing and Linking Cartographic Resources across Discovery Environments
}

\author{
Kimberly Durante ${ }^{\mathrm{a}, *}$, Emily Prince ${ }^{\mathrm{b}}$ \\ a Stanford University Libraries, kdurante@stanford.edu \\ ${ }^{b}$ Stanford University Libraries, ebprince@stanford.edu \\ * Corresponding author
}

Keywords: Georeferenced maps, Digital preservation repositories, Metadata

\begin{abstract}
:
This presentation will highlight Stanford University Libraries' development of institutional best practices for identifying relationships among cartographic resources stored within the university's digital preservation repository and made available to end users from contextualized discovery catalogs: SearchWorks and EarthWorks. The use of map interfaces as a tool for searching and retrieving of geographic information has greatly influenced the design of structural metadata and the related management of elegant workflows for supporting consistent procedures, both amongst the cartographic metadata librarians, as well as across greater Stanford Library policies.
\end{abstract}

As collections of maps, atlases, and geospatial imagery are increasingly being digitized and georeferenced for use in a number of research contexts and purposes, new digital data are produced and made available through spatial data infrastructures, geoportals, and programmatic APIs. Our goal is to preserve the lineage that exists between primary source materials - such as centuries-old maps - and their derivative or versioned datasets, and to make this track both transparent and easily actionable for end users who are evaluating materials found through search engines or contextualized discovery platforms. The goal is to provide users with easily navigable links between the source materials and all subsequent versions.

Our work relies on the use of structured metadata to define relationship types that commonly exist between cartographic materials old and new, and includes the use of persistent links that direct users to both source materials, as well as to georectified imagery, or shapefile index maps that are based upon those source materials. Using examples of Japanese maps of Tokyo, from several of Stanford's rare and historic cartographic collections (housed at the East Asia Library, David Rumsey Map Center, and Branner Earth Sciences Library), we will present a standardized list of relationships which are prevalent among cartographic data collections and we will demonstrate how we have designed and leveraged metadata in these cases in order to facilitate the user's intuitive discovery, reuse, and citation of related map content. 